\title{
AUTOMATION OF HUMAN DECISION PROCESSES FOR ROUTE COMPLETION FOR AIRSPACE DESIGN ANALYSIS
}

\author{
Lisa A. Schaefer \\ Department of Mathematics and Statistics \\ American University \\ 4400 Massachusetts Avenue NW \\ Washington, D.C. 20016, U.S.A.
}

\begin{abstract}
This paper describes an algorithm for approximating missing data in air traffic routes thereby allowing the lengths of different routes to be compared for our simulation analyses. We were given air traffic routes that had origin, destination, and complete route information inside of the problem design area. Analyst judgment was necessary for completing the routes outside of the design area. We automated the analyst decision processes so that comparisons could be made across scenarios. This process was required for thousands of routes. We analyze design performance measures and show that the resulting distances among the redesigned routes differ from baseline route lengths.
\end{abstract}

\section{BACKGROUND}

Airspace design has become more prevalent over the past several years (FAA 2003). After airline deregulation, airlines developed hub-and-spoke systems and reduced fares, which resulted in many more flights than ever before. Since then congestion has increased; therefore, airspace needs to be designed with more efficiency in mind, while maintaining safety standards.

As the National Airspace System (NAS) continues to evolve, airspace will need to be redesigned. New technology such as radio navigation (Becher and Formosa 2000) will allow aircraft to fly different routes, possibly resulting in congestion shifting to different geographical areas. Reduced vertical separation minimums (McFarland and Maroney 2001) will create more flight levels, possibly making it desirable to stratify sectors at different levels. Continual changes in demand patterns over time, due to population and economic change, (Bhadra 2003) may increase congestion in some areas while reducing demand in other areas.

A major effort within the airspace design process is creating the pattern of routes that aircraft will fly. Designing routes is an iterative process that requires buy-in from several affected parties, such as air traffic controllers, airlines, and citizens who may be affected by noise from overhead aircraft. A large part of MITRE's role in such a process is to facilitate agreement among the affected parties by providing an unbiased analysis of design options. Airspace design scenarios are usually evaluated with several different performance measures, such as delay, controller workload, noise levels, and route length (Hoffman et al. 1999). In this paper we focus on methods for comparing route lengths among several airspace design scenarios.

We developed heuristics to extend routes within the design area to airports beyond the design area. This heuristic can be used for future airspace design projects to assist in route design and route-length comparisons among different airspace design scenarios.

\section{PROBLEM DESCRIPTION AND APPROACH}

Working with MITRE, an air traffic controller team designed sets of routes for aircraft arriving to or departing from airports within the metropolitan area under redesign. Several thousand routes were developed. The routes developed by the controllers were very detailed within the design area, but this level of design detail does not extend for the full length of the routes. Therefore we needed to complete the routes outside of the design area. We developed a method to automate the completion of these routes.

We developed a process for testing and evaluating our automation method. We created an example problem for a hypothetical metropolitan area for experimenting with our rules. The metro area has several airports. We developed an appropriate As-Is route structure, similar to the design of routes in the current NAS, to represent a baseline for today's routes for our artificial metro area.

We designed two alternative scenario route structures for our test example. The scenarios were based on reasonable attempts to organize the air traffic flow and were critiqued by several air traffic controllers. Scenario 1 is a slight change to the existing route structure. Scenario 2 represents a major change to the existing route structure.

We then analyzed the two Scenarios and compared their route lengths to the As-Is design. 


\section{SCENARIO DESCRIPTIONS}

The air traffic controller team selected two different approaches for generating reroutes for the alternative scenarios. For each scenario, the airspace designs covered different areas.

Scenario 1 is a slight change to the existing route structure. The design area for Scenario 1 extends approximately 150 miles south, 130 miles north, 130 miles east, and 100 miles west of the metro area. Scenario 2 represents a major change to the existing route structure. The design area for Scenario 2 extends approximately 300 miles south, 200 miles north, 200 miles east, and 200 miles west of the metro area. Figure 1a shows the departure and arrival routes for Scenario 1. Figure $1 \mathrm{~b}$ shows the departure and arrival routes for Scenario 2. Many routes extend from the tips of the design shown in Figures $1 \mathrm{a}$ and $1 \mathrm{~b}$.

Figure 2 shows an example of the waypoints and geographic shapes of departure routes from the metro area to a typical airport for the As-Is scenario, Scenario 1 and Scenario 2. The routes for Scenarios 1 and 2 are incomplete since they only extend to the edge of the redesign, not all the way to Airport A. The As-Is routes are outlined in bold. The Scenario 1 routes are outlined with thin solid lines. Scenario 2 is represented by dashed lines. In this case, the departure route for Scenario 1 (in the middle of Figure 2 a) is more direct than the route for the As-Is scenario (on the right). The route for Scenario 2 (on the left) is the most direct of the three. This is not generally true for all airport pairs. Some new routes are longer than the As-Is scenario to route around congestion during peak demand periods.
Figure $2 \mathrm{~b}$ shows an example of the geographic shapes of arrival routes from the metro area to Airport A for the As-Is scenario, Scenario 1, and Scenario 2.

Scenario 1 represents a small change from the As-Is scenario in which the routes use most of the same fixes and only deviate slightly, in this case a few miles to the west, from the As-Is scenario. Scenario 2 is a major redesign, very different than the As-Is scenario in that they separate from the original route a significant distance from the metro area.

\section{METHOD}

To determine routes for the As-Is scenario, we identified common routes for relevant airport pairs in Enhanced Traffic Management System (ETMS) flight plan data.

It is possible to compare the distances of the three routes in Figures $2 \mathrm{a}$ and $2 \mathrm{~b}$ starting from the edge of the design area. It is also possible that a completed path could be drawn directly from the last scenario point on the edge of the design area to Airport A. However since the redesign was only intending to change the routes within the design area, we adopted the policy that routes must match outside the design area when comparing route lengths. This is because air traffic controllers outside the design area are not expected to route traffic differently due to the new design plans.

We developed a set of methods for conducting route length analyses to ensure that routes share a common path outside the design area for arrival routes in the As-Is scenario, Scenario 1, and Scenario 2. It was necessary for the
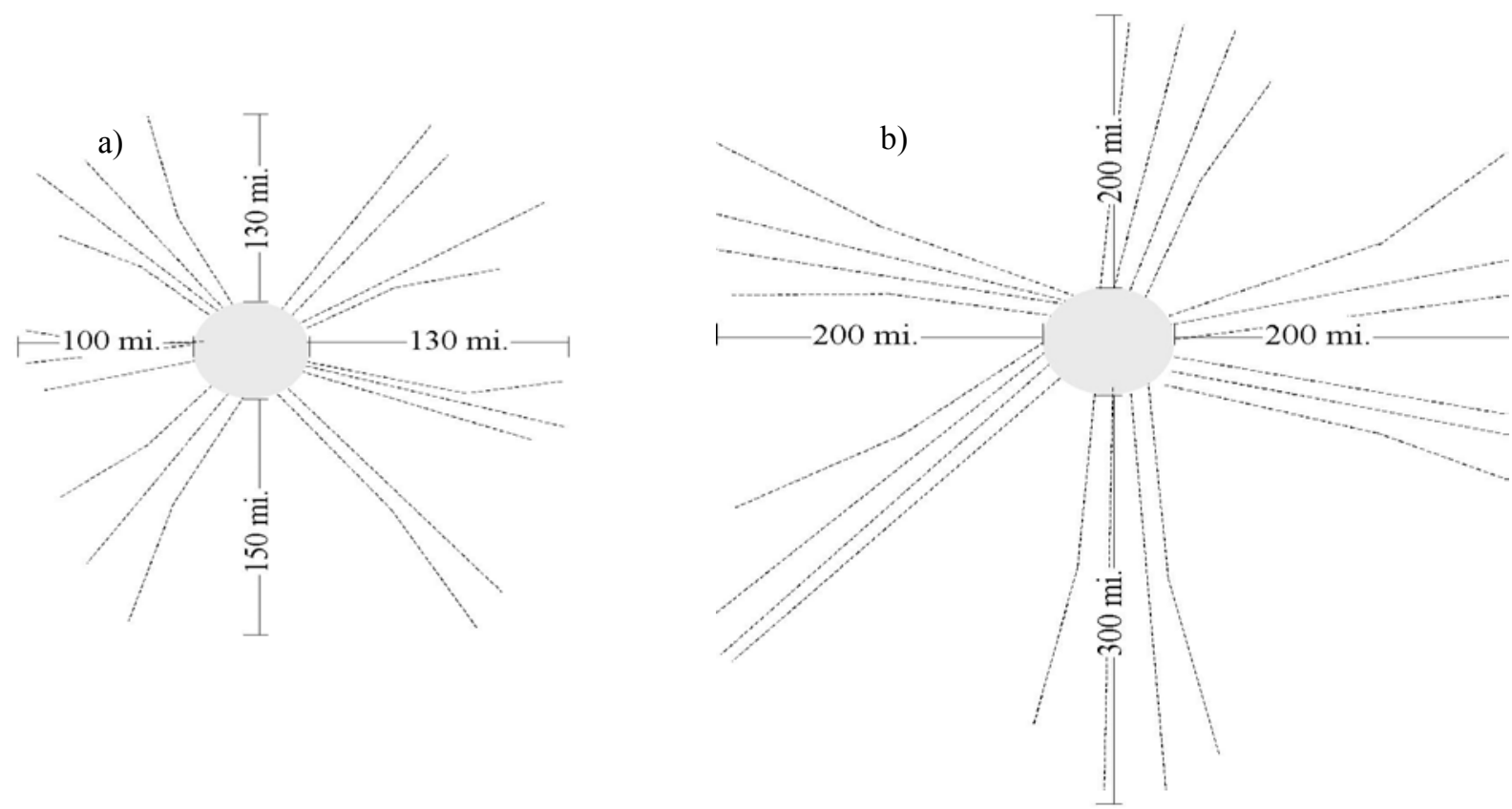

Figure 1a): Organized Departure and Arrival Routes within the Design Area for Scenario 1; 1b): Organized Departure and Arrival Routes within the Design Area for Scenario 2 

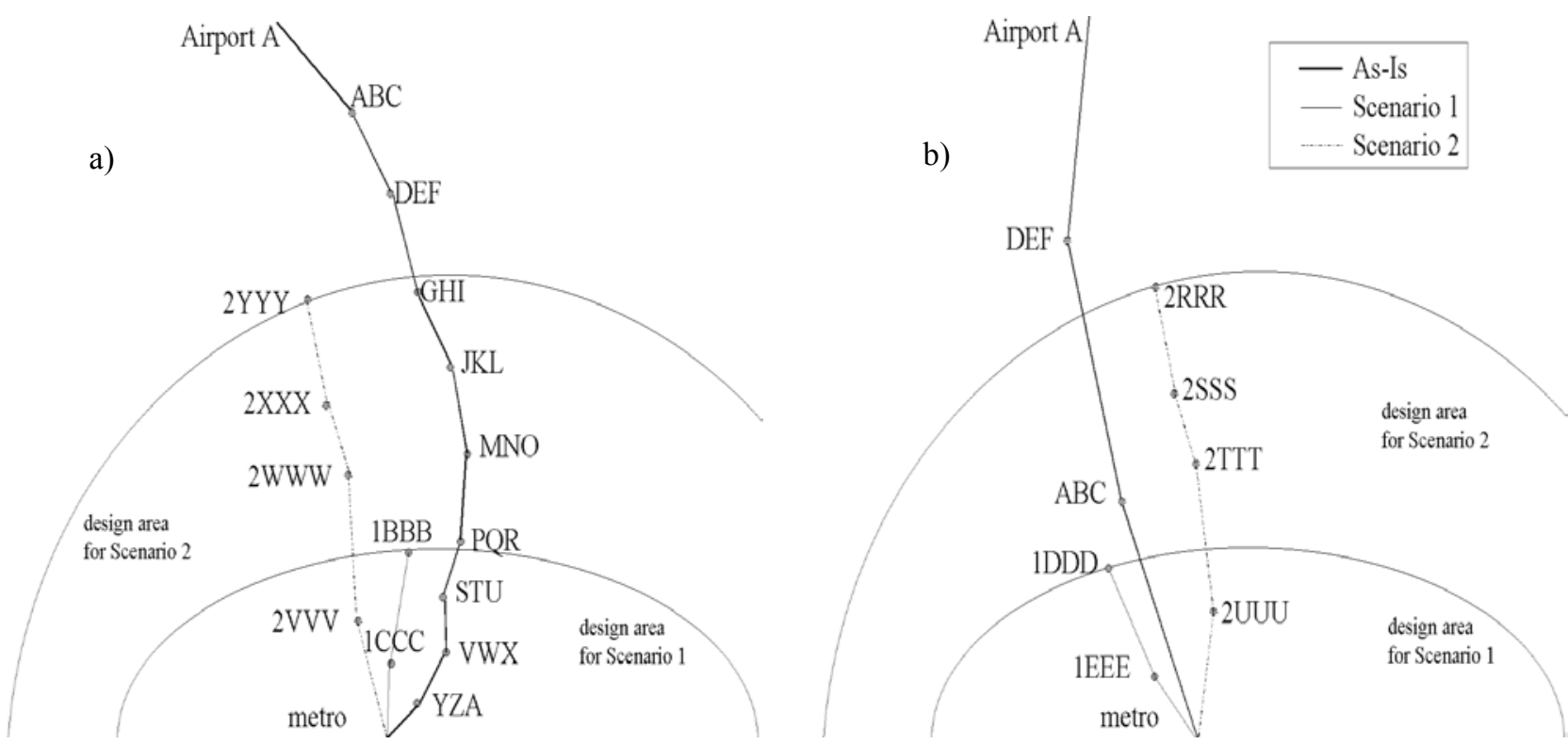

Figure 2a): Departure Route from Metro Area to Airport A for As-Is, Scenario 1, and Scenario 2; 2b): Arrival Route from Airport A to Metro Area for As-Is, Scenario 1, and Scenario 2

waypoints outside the design area for each of the scenarios to match identically to each other so route lengths could be properly compared. There were four cases that could describe a set of routes for a given airport pair. Algorithms were developed to fulfill each of the cases. The cases, along with their corresponding algorithms, are listed in Figure 3. Example route pairs, from the As-Is scenario and one of the new scenarios, corresponding to each of the cases are shown in Figures 4a through 4d respectively.

The algorithms are described as appropriate for arrival routes. Departure routes were handled similarly, except the order of the waypoints was reversed. Figure 4 shows a graphical example of each of the cases in Figure 3 within the context of a scenario. The As-Is routes are in bold.

Figure $4 \mathrm{a}$ shows that Airport $\mathrm{W}$ is within the area where routes are redesigned for Scenario 2. Figure $4 \mathrm{~b}$ shows that waypoint KKK is used in both the As-Is and Scenario routes for the given airport pair. Figure $4 \mathrm{c}$ shows that the As-Is and Scenario routes have waypoints near each other in the vicinity of entering the design area. Figure $4 \mathrm{~d}$ shows that As-Is and Scenario routes do not have waypoints near each other in the vicinity where they enter the design area.

For each airport pair, the cases are examined in order until we find one that applies to the As-Is and scenario routes. For Case 1 we check if the origin airport is within the design area. If so, we merely need to ensure that the route was complete from the departure airport to the destination airport in the metro area.

If the origin airport is not within the design area, we used the next simplest algorithm. The algorithm for Case 2 checks if the first waypoint of the route within or near the design area is at the same location as one of the waypoints

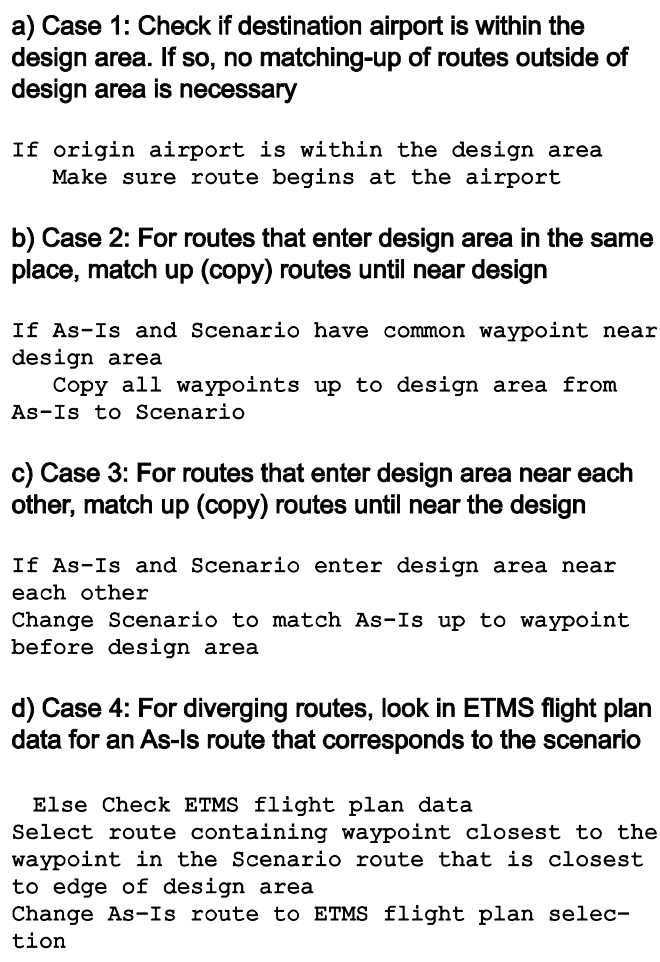

c) Case 3: For routes that enter design area near each other, match up (copy) routes until near the design

If $\mathrm{As}-$ Is and Scenario enter design area near each other

Change Scenario to match As-Is up to waypoint

before design area

d) Case 4: For diverging routes, look in ETMS flight plan data for an As-Is route that corresponds to the scenario

Else Check ETMS flight plan data

Select route containing waypoint closest to the

waypoint in the scenario route that is closest

to edge of design area

Change As-Is route to ETMS flight plan selec-

tion

Figure 3: Route Comparison Cases with their

Algorithms

in the As-Is scenario. We defined "near the design" as within 20 miles of the endpoint of a designed route segment farthest from the metro area.

Case 3 is somewhat more complex than Case 2, which simply looks for identical waypoints as routes enter the de- 


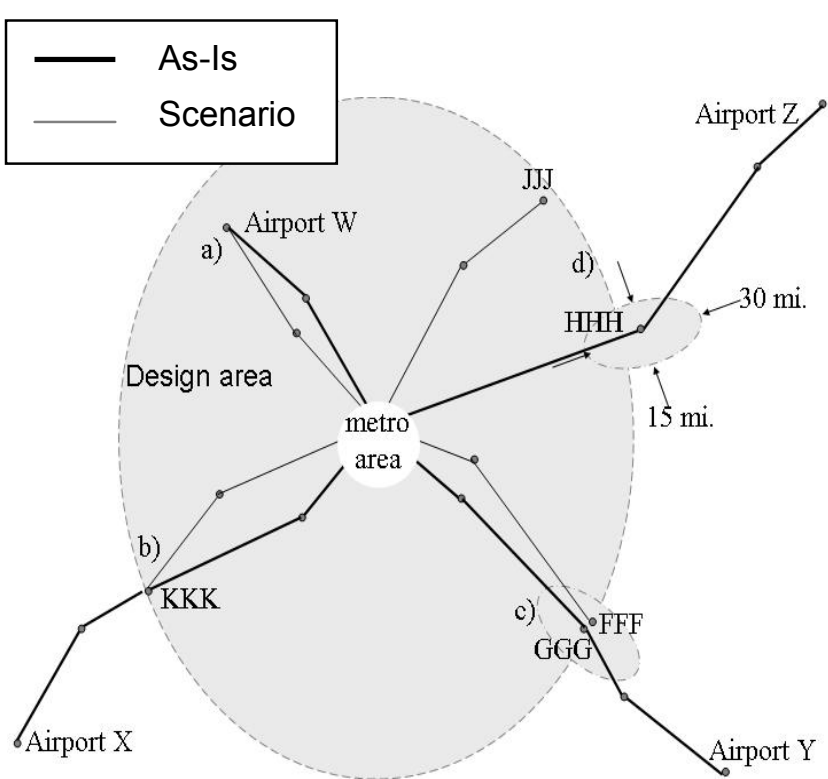

Figure 4a): Airport within Design Area; 4b): As-Is and Scenario Have Common Waypoint Near Design Area; 4c): As-Is and Scenario Have Waypoints Near Each Other at Edge of Design Area; 4d): As-Is and Scenario Do Not Have Waypoints Near Each Other at Edge of Design Area

sign area. For Case 3, one must determine if waypoints are near each other and near the edge of the design area at the same waypoint on their route. We define "near each other" as within 15 miles of each other perpendicular to the direction to the metro area and within 30 miles of each other parallel to the direction to the metro area. This was used for waypoints at the segment of the route closest to the start of a designed route segment. A picture of this definition is included in Figure 4d.

For the routes that did not match the criteria for Cases 1 through 3, we developed Case 4, which required referring to ETMS flight plan data to find a route near the designed route for the scenario. Although the metropolitan area is hypothetical, we used ETMS route data for airports having similar geographical characteristics to our metropolitan area so that our As-Is scenario would closely approximate the routes of the current system.

Since Scenario 1 is only a slight variation on today's established routes, we only needed Cases 1 and 2 to match most of Scenario 1 routes to As-Is routes. However, Scenario 2 is a major redesign, so we were required to find current routes other than the standard set for some As-Is routes to be able to make a reasonable comparison. Thus we were required to refer to ETMS flight plans for some routes. This required that we also change the Scenario 1 routes to ensure that all three sets of routes are comparable to each other.

With good knowledge of airport and navaid locations, we browsed the routes and determined that Case 4 affected too few routes, approximately 200 routes, to justify coding an ETMS lookup, which would be a large effort. Rather, manually adjusting the routes affected by that rule was a more efficient method for calibrating routes.

We did not develop extra routes to consider effects of weather and congestion. Weather effects are so variable that it is unclear how much knowledge would be gained by analyzing any particular additional route. Congestion was accounted for in a separate effort that analyzed delay.

The Total Airport and Airspace Model (TAAM) is a simulation model that determines delays and other performance measures for airport and airspace designs. To calculate route length and to test the routes as input for our simulation analyses, the routes were used as input for a TAAM run. The output was analyzed in MapInfo, a Geographic Information System to calculate route lengths. The differences were analyzed with Excel.

\section{RESULTS}

Routes flown by TAAM were displayed in MapInfo and TAAM's IDIS graphical display for validation. Table 1 lists the routes from Airport A to the metro area for the AsIs, Scenario 1, and Scenario 2 before and after the algorithms were applied to the routes. In this case, the algorithm for Case 3 was executed on the route for Scenario 1 since Airport A is outside the design area and the route for Scenario 1 does not share a common waypoint with the AsIs route at the edge of the design area. For Scenario 1, waypoint $1 \mathrm{BBB}$ is near waypoint $\mathrm{PQR}$ of the As-Is route. Thus we matched the route for Scenario 1 up to waypoint $\mathrm{PQR}$ and joined the original route for Scenario 1 at $1 \mathrm{CCC}$, the first waypoint within the design area for Scenario 1.

Algorithm 4 was executed on the route for Scenario 2. There were no waypoints along the route for Scenario 2 near waypoint GHI. Thus we looked up routes in ETMS

Table 1: Before-and-After Routes from Airport A to our Hypothetical Metro Area for As-Is Case, Scenario 1 , and Scenario 2

\begin{tabular}{|c|l|lll|}
\hline Scenario & Analysis Stage & Route & & \\
\hline As-Is & Before & AirportA & ABC DEF GHI JKL MNO PQR STU VWX YZA metro \\
\hline & After & AirportA ZXY WVU GHI JKL MNO PQR STU VWX YZA metro \\
\hline 1 & Before & AirportA 1BBB 1CCC metro & \\
\hline & After & AirportA & \\
\hline 2 & Before & AirportA & 2YYY 2XXX GHI JKL MNO PQR 1CCC metro \\
\hline & After & AirportA ZXY WVU 2XXX 2WWW 2VVV metro \\
\hline
\end{tabular}


for Airport A to the metro area. We found a route that went through waypoints ZYX and WVU that came closer to the route for Scenario 2. Thus we changed the beginning of the routes for As-Is, Scenario 1, and Scenario 2 to be able to properly compare route lengths. Figure 5 shows the difference in geographical shape of routes from Airport A to the metro area after the routes were matched up. The As-Is route is outlined in bold. The routes after the scenarios branch away from the As-Is route is outlined with thin solid and dashed lines, respectively. For clarity, the original shape before our algorithms were executed, which was shown in Figure 4, is included in Figure 5a.

The key performance measure we needed to obtain from the routes was the difference in length among the

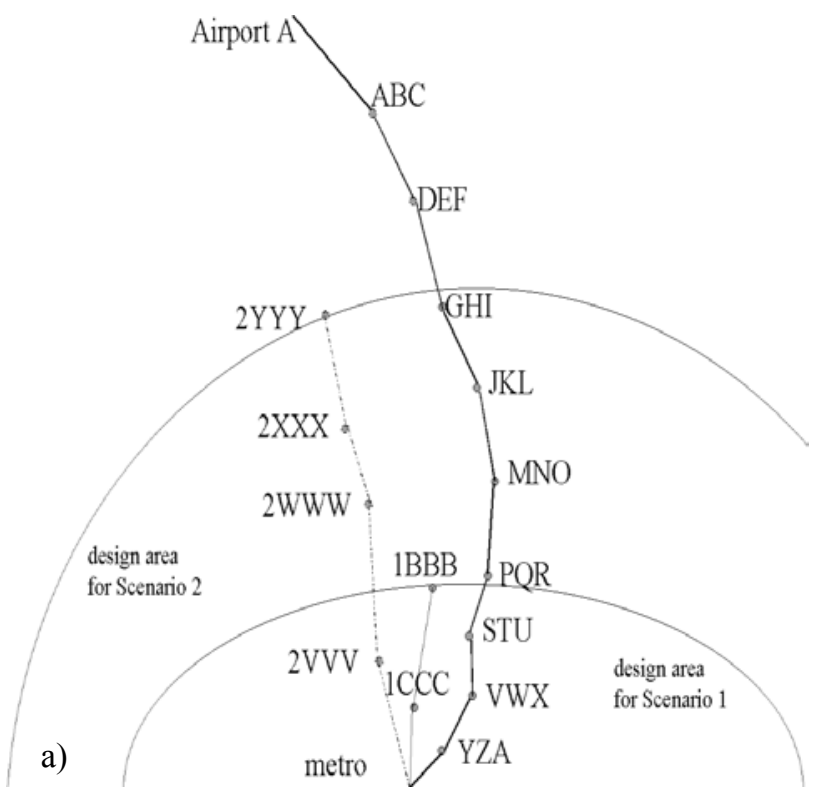

routes Figure 6 shows a histogram of the differences in the route lengths for the 1612 routes. A positive difference means that the route length of the scenario was longer than the route for the As-Is case. Figure 7 shows a percent change in length of the routes.

The results in Figure 6 signify that most routes had little change to their length; however, a few routes were affected by the redesign by more than 50 miles. Figure 7 shows that route length differences were somewhat proportional to original route distance. Some routes increased because the design spread routes out to prevent congestion during peak demand periods.

Figure 5: Difference in Geography of Routes from Airport A to Metro Area a) Before and b) After Matching Up Routes Outside Design Area

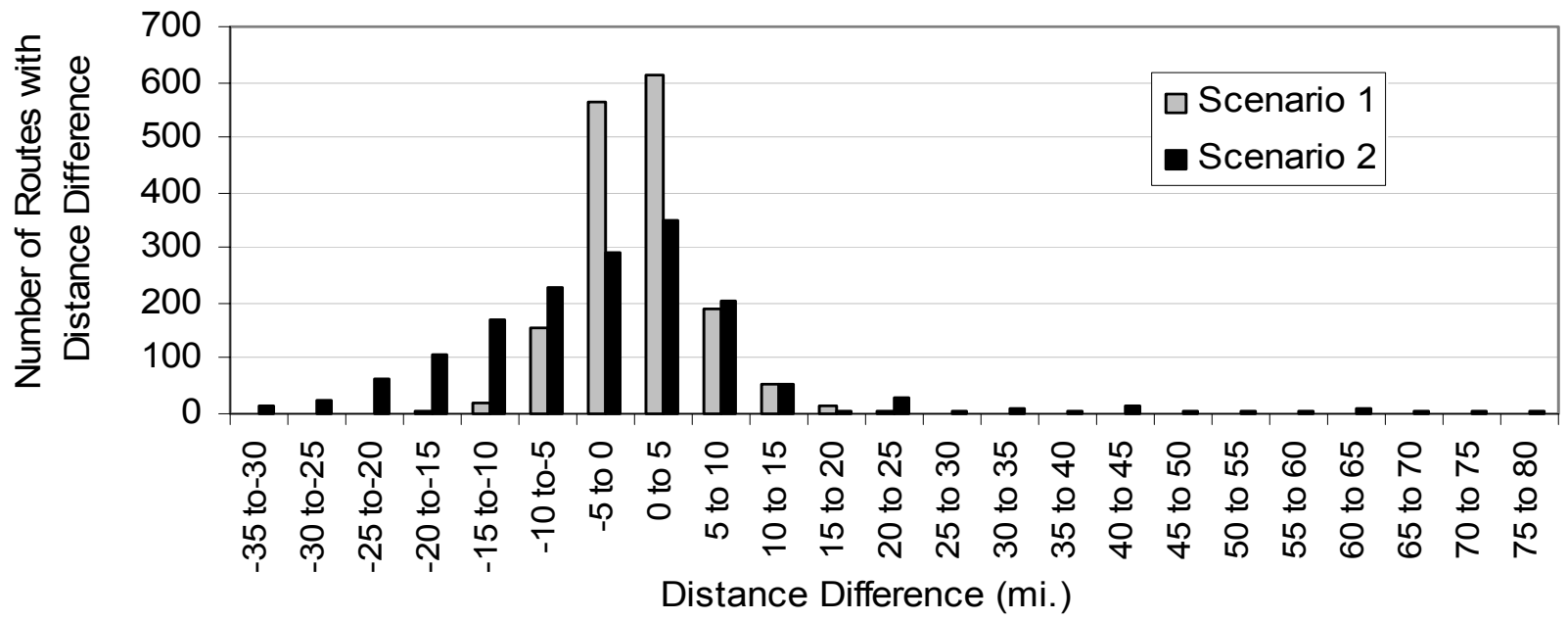

Figure 6: Number of Routes with Length Differences by 5-Mile Bins for Scenarios 1 and 2 


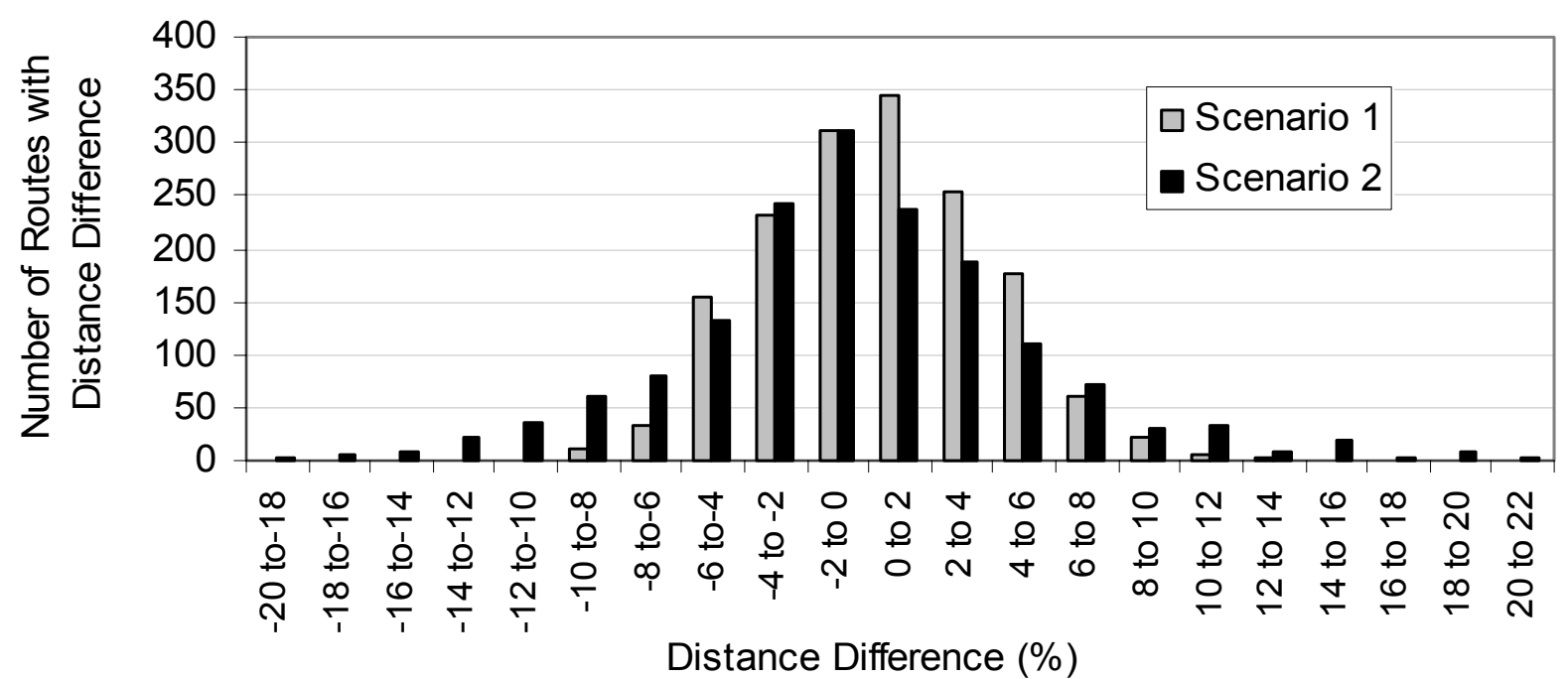

Figure 7: Number of Routes with Length Differences by 2\% Bins for Scenarios 1 and 2

\section{CONCLUSION}

It was difficult to quantitatively describe how to complete the routes for different scenarios. We needed to connect the routes at some reasonable point before the design area to be able to properly compare route lengths. It was not obvious for most routes where this point should be. The four cases that we developed worked well to help us create routes that could be reasonably compared for differences in length due to new airspace designs.

Airspace redesign projects introduce a more organized structure to air traffic. This results in lower controller workload and increased throughput, possibly at the expense of route length. Even though many routes for stable weather conditions are longer, the need for ground delays and vectoring is reduced, resulting in decreased arrival delay. Most of the savings for this redesign are realized during poor weather conditions and during periods of heavy congestion.

\section{REFERENCES}

Becher T. and J. Formosa. 2000. RNAV near-term terminal procedures development. In 3rd USA/Europe Air Traffic Management R\&D Seminar, Napoli.

Bhadra D. 2003. Demand for air travel in the United States: Bottom-up econometric estimation and implications for forecasts by origin-destination pairs. Journal of Air Transportation 8 (2).

Federal Aviation Administration (FAA). 2003. National airspace redesign. Available online via $<$ http: //www . faa.gov/ats/nar/> [Accessed July 8, 2004].

Hoffman, J., T. Abrahamsen, S. Bowden, T. Brothersen and D. Moch-Mooney. 1999. Initial eastern united states airspace problem identification. MITRE Technical Report 99W0000032.
McFarland A. and D. Maroney. 2001. Eliminating the altitude-for-direction rule implementing reduced vertical separation minimum in the U. S. MITRE Paper MP01W0000167.

\section{ACKNOWLEDGMENTS}

The author would like to thank Thor Abrahamsen, Joe Hoffman, and Debby Pool for their comments toward this paper.

\section{AUTHOR BIOGRAPHY}

LISA A. SCHAEFER is a Visiting Assistant Professor in the Department of Mathematics and Statistics at American University in Washington, D.C. and a Senior Simulation Engineering consultant at the MITRE Corporation in McLean, Virginia. She received her B.S.E. and M.S. in civil / transportation engineering and Ph.D. in industrial engineering at Arizona State University. Her interests include future concepts for transportation and simulation concepts for mainstream usage. Her email and web addresses are: <LSchaeferAZ@hotmail.com> and http://academic2 american. edu/ schaefer

This work was produced for the U.S. Government under Contract DTFA01-01-C-00001 and is subject to Federal Aviation Administration Acquisition Management System Clause 3.5-13, Rights In Data-General, Alt. III and Alt. IV (Oct., 1996).

The contents of this document reflect the views of the author and The MITRE Corporation and do not necessarily reflect the views of the FAA or the DOT. Neither the Federal Aviation Administration nor the Department of Transportation makes any warranty or guarantee, expressed or implied, concerning the content or accuracy of these views.

(C) 2004 The MITRE Corporation 